\title{
AN EXAMINATION OF THE EFFICACY OF A BRIEF EDUCATIONAL PROGRAM ON DRIVER DISTRACTION
}

\author{
Arthur F. Kramer \& Jason S. McCarley \\ Beckman Institute \\ University of Illinois \\ Urbana-Champaign, IL USA \\ E-mail: akramer@s.psych.uiuc.edu \\ Scott P. Geisler \\ General Motors \\ North American Engineering \\ Warren, MI USA \\ E-mail: scott.p.geisler@gm.com
}

\begin{abstract}
Summary: A study examined the influence of a brief educational intervention on self-reports of past and intended future distracted driving behavior, and on perception of the danger of various driver distractions (e.g. map reading, grooming, etc). Respondents completed a series of rating scales, reporting (A) the frequency with which they had participated in various distracting activities while driving in the past, (B) the frequency with which they expected to participate in those activities in the future, and (C) the perceived danger of those activities. Half of the respondents completed the rating scales after first watching a series short animated videos from the driver education program. The remaining respondents (controls) completed the surveys prior to viewing the video segments. As compared to the control subjects, participants who viewed the video segments prior to filling out the rating scales reported reliably higher levels of perceived danger for a number of distracting activities. Video segments did not, however, appear to affect respondents' anticipated future behavior.
\end{abstract}

\section{INTRODUCTION}

As cellular phones, navigation systems, and other forms of in-vehicle technology have become more popular, concerns about their potential distracting effects on drivers, have increased. A number of studies have suggested that in-vehicle technologies, even when designed to minimize manual or sensory interference, can divert cognitive resources from a primary task, disrupting various aspects of driving performance (e.g., Strayer \& Johnson, 2001). Accordingly, use of invehicle technologies has been shown to be associated with an increased likelihood of accidents (e.g., Redelmeier \& Tibshirani, 1997).

Given the economic and personal benefits that in-vehicle technologies convey, however, it may be neither feasible nor desirable to proscribe their use. An alternative approach to reducing invehicle distraction may be to ensure that drivers are aware of the risks that distracting activities present, and to encourage drivers to self-regulate their participation in such activities while driving. The goal of the present study was to assess the efficacy of a brief educational intervention on drivers' perceptions of and intentions to engage in various distracting activities. 
Toward that end, educational materials and a survey were delivered to participants over the internet. A between-groups experimental design was used to examine the effects of the intervention on survey responses. Survey items included questions about activities both related and unrelated to in-vehicle technology.

\section{METHOD}

\section{Participants}

Participants were 1,423 respondents ( 643 female, mean age $=46$ years, minimum $=20$ years, maximum $=60$ years) contacted via e-mail to participate in an online study. All participants were drivers who had been licensed for at least a year.

\section{Educational Materials}

Respondents were randomly sorted into experimental and control groups $(n=733$ and $n=690$, respectively). All respondents completed an online survey on the topic of driver distraction. Before completing the survey, participants in the experimental group viewed a brief (1-2 minutes each) set of animated videos meant to discourage distracted driving. Videos included introductory and concluding segments, and six segments focused on more specific topics: eating while driving, tending to children while driving, reading a map while driving, using a phone while driving, adjusting the radio while driving, and the effects of brief response delays on braking distance. Each participant in the experimental group saw the introductory segment, three randomly chosen segments on specific topics, and the concluding segment. Participants in the control group completed the survey without viewing the educational materials.

\section{Surveys}

In addition to demographic questions, surveys comprised a total of 36 items concerning 12 potentially distracting activities: dialing a hand-held cellular phone, talking on a hands-free cellular phone, talking on a hand-held cellular phone, talking to a passenger, tending to a child, eating, drinking a non-alcoholic beverage, reading a newspaper or book, reading a map, changing a CD or tape, changing the radio station, grooming (e.g., brushing hair, applying makeup). Survey items 1 through 12 asked participants to estimate how frequently they had engaged in each of the distracting activities while driving in the past month. Items 13 through 24 asked participants to estimate how frequently they would participate in each of the specified activities while driving in the upcoming month. Items 25 through 36 asked participants to estimate how dangerous they perceived each of the specified activities to be. Responses for items 1 through 24 were made on a five-point Likert scale anchored with the labels "Very infrequently" and "Very frequently." Responses for items 25 through 36 were made on a fivepoint Likert scale anchored with the labels "Very dangerous" and "Very safe." 


\section{RESULTS}

\section{Past and Anticipated Frequency of Distracted Driving}

Figure 1 presents mean reported frequency ratings for the twelve activities of interest, collapsed across age, gender, and time frame. For omnibus analysis, data were grouped according to age of respondent (20-29, 30-39, 40-49, 50-59, 60-69) and were submitted to a mixed ANOVA with condition (experimental or control), gender, and age group as between-subject variables and with activity (e.g., dialing a hand-held phone, talking on a hand-held phone) and time frame (previous month or next month) as within-subjects variables. P-values reported below are uncorrected. All effects described as reliable, however, remained significant after Greenhouse-Geisser correction.

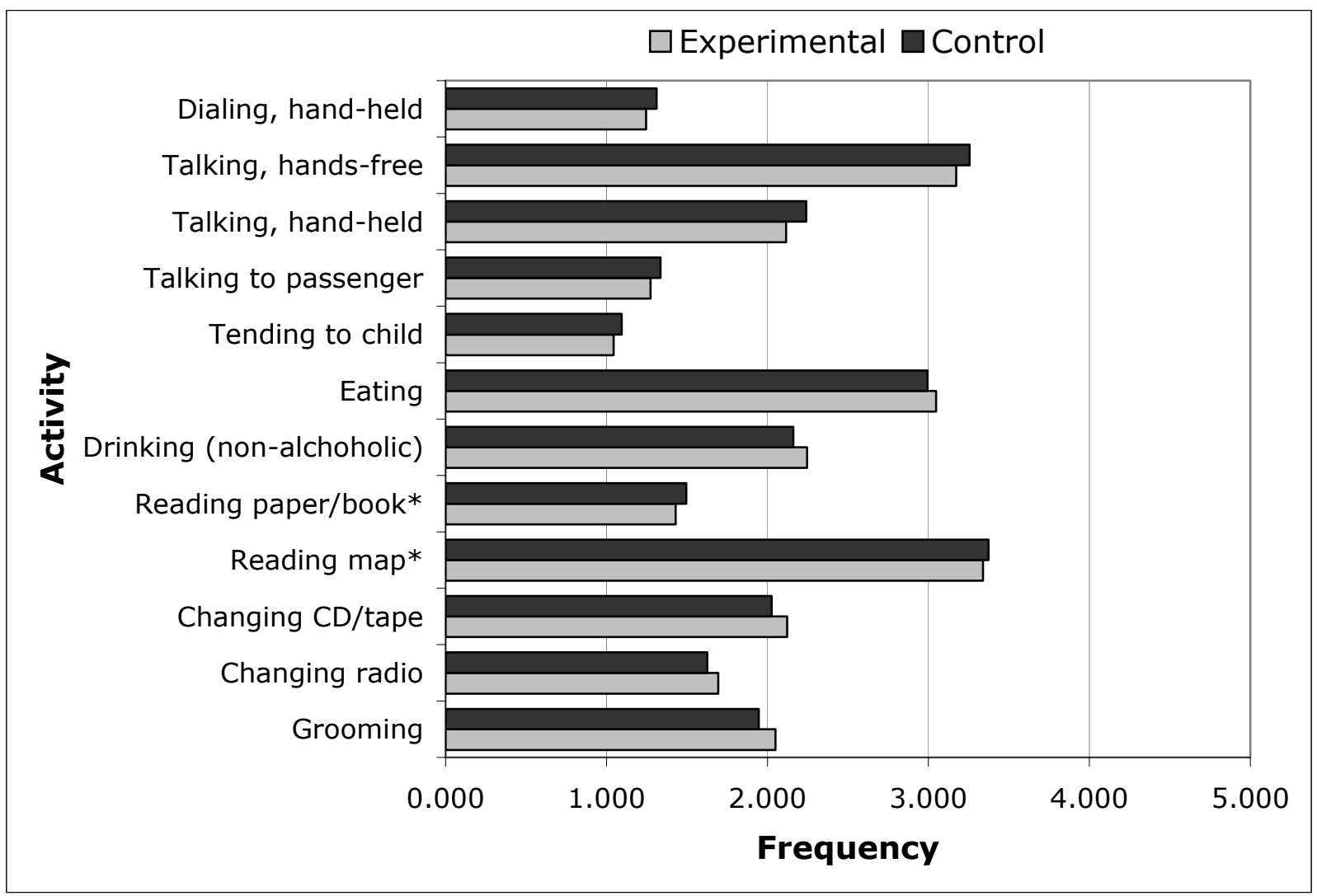

Figure 1. Mean frequency ratings for each of twelve activities, collapsed across age, gender, and time frame (last month vs. next month). Asterisks indicate significant differences between experimental and control groups. These differences, however, were independent of time frame, suggesting that the educational materials did not selectively reduce the anticipated future frequency of distracted driving.

Reliable main effects of gender and age indicated that past and anticipated frequency of engaging in distracting activities while driving was higher in general for men than for women, $p=.031$, and that the frequency of distracted driving tended to decrease with respondent age, $p<.001$. A reliable main effect of activity indicated, not surprisingly, that some distracting activities were more frequent than others, $p<.001$. Reliable interactions of activity by age, $p<.001$, activity by gender, $p<.001$, and activity by age by gender, $p<.05$, indicated further that the frequency of 
various distracting activities varied across demographic groups. Of primary interest were the effects of the educational intervention on reported past and anticipated frequencies of various distracting behaviors. In particular, a finding that the anticipated future frequency of engaging in distracting activities while driving was selectively reduced for participants in the experimental group would provide evidence for the effectiveness of the intervention. Analysis revealed no reliable main effect of condition, $p=.145$, but did produce a reliable interaction of condition with activity, $p=.010$, reflecting the finding that the experimental intervention selectively modulated the overall (past and anticipated) reported frequencies of some activities. Post-hoc ttests revealed more specifically that respondents in the experimental group reported reliably lower overall frequencies of reading a book/newspaper and reading a map while driving, $p \mathrm{~s}<$ .05. Crucially, however, these changes were independent of time frame, and did not differ significantly for reports of past and anticipated future behavior; analysis indicated a nonsignificant two-way interaction of condition by time frame, $p=.303$, and produced no reliable higher way interactions involving those factors, all $p s>.15$. In other words, the intervention did not appear to selectively decrease experimental respondents' anticipated future participation in dstracting activities while driving. Between-group differences in overall reported frequencies may reflect a response to perceived experimenter demand.

\section{Perceived Danger of Distracting Activities}

Figure 2 presents mean danger ratings for the twelve activities of interest, collapsed across age and gender. For omnibus analysis, data were again grouped according to age of respondent (2029, 30-39, 40-49, 50-59, 60-69) and were submitted to a mixed ANOVA with condition (experimental or control), gender, and age group as between-subject variables and with activity (e.g., dialing a hand-held phone, talking on a hand-held phone) as a within-subjects variable.

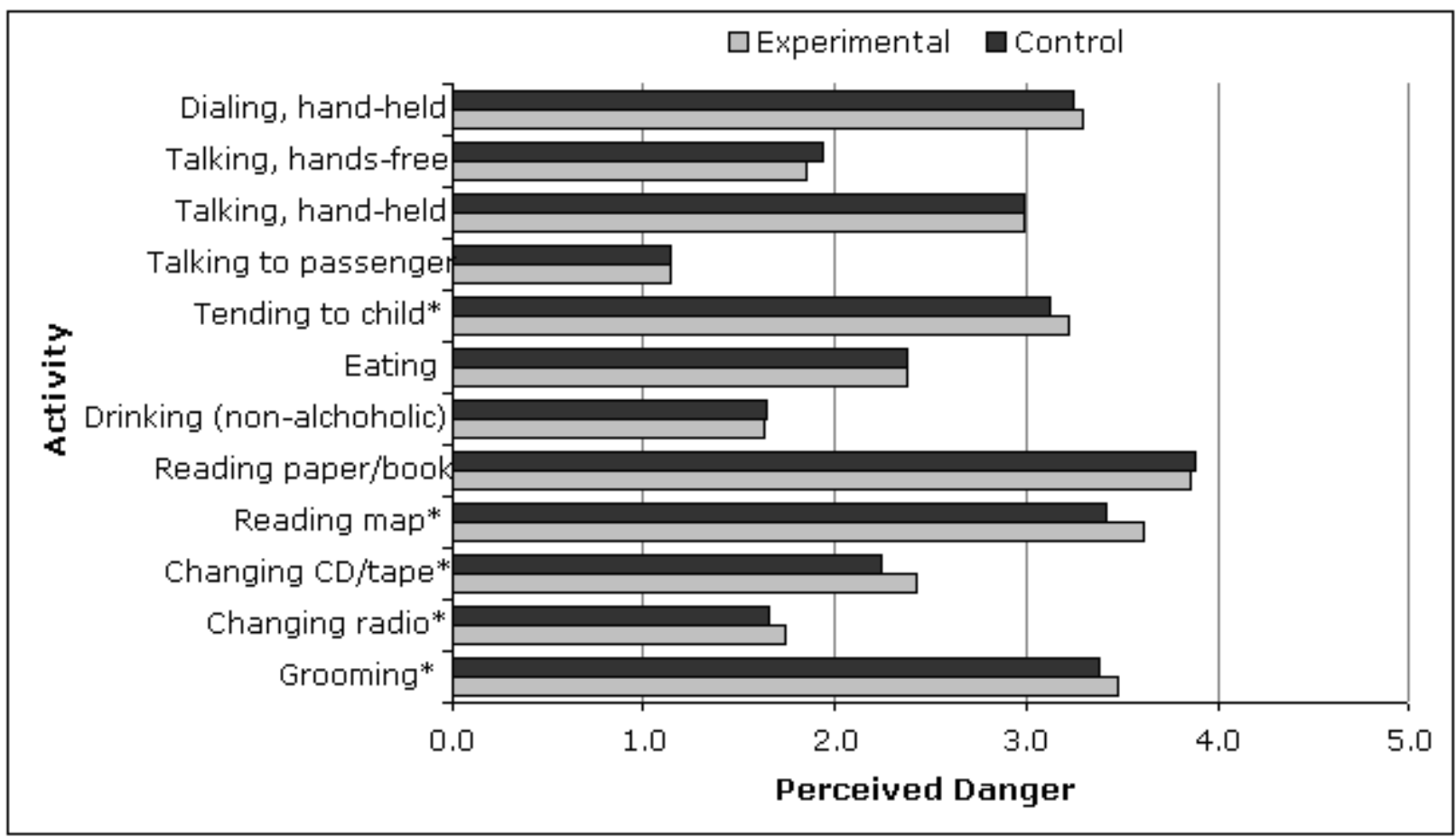

Figure 2. Mean danger ratings for each of twelve activities, collapsed across age and gender. Asterisks indicate significant differences between experimental and control groups. 
Reliable main effects of age and gender, $p s<.001$, indicated that the perceived danger of distracting activities increased with respondent age, and were generally larger for women than for men. A reliable main effect of activity, $p<.001$, reflected the fact that some activities were considered more dangerous than others, though reliable interactions of activity by age and activity by gender indicated that the danger of various activities varied across demographic groups, $p s<.001$. Of foremost importance were effects involving experimental condition. Analysis revealed no reliable main effect of condition, $\mathrm{p}=.090$, but did produce a reliable interaction of condition by activity, $\mathrm{p}<.001$. Post-hoc t-tests found five which were reliably rated as being more dangerous by the experimental group than by the control groups, all $p \mathrm{~s}<.05$ : tending to a child, reading a map, changing a CD or tape, changing the radio station, and grooming.

\section{DISCUSSION}

The present study examined the efficacy of a set of brief educational videos in modifying respondents' perceptions of and intentions to engage in distracting activities while driving. Data gave no evidence of a selective decrease in experimental group respondents' estimated frequency of future distracted driving, suggesting that the materials tested did little to change respondents' behavioral intentions. Results did, though, indicate a selective but reliable effect on reported perceptions of the danger of in-vehicle distractions; experimental group respondents assessed a number of activities as being more dangerous than did control group respondents. It is possible that the effect of the experimental manipulations on assessments of danger was no more than a response to perceived experimenter demands. Alternatively, the data may indicate a true dissociation between respondents' beliefs about the risks of driver distraction and their own intentions to avoid distracted driving. This pattern of results might reflect a form of overconfidence bias. That is, respondents might recognize the risks of distracted driving in general, but overestimate their own ability to contend with those risks. Indeed, overconfidence has frequently been implicated as a contributor to risk-taking behavior in driving and other applied domains (e.g., Goh \& Wiegmann, 2001; Matsuura, Ishida \& Ishimatsu, 2002). Future research will be necessary to determine whether efforts to reduce overconfidence might also reduce drivers' intentions of driving under distraction, and as importantly, to determine whether such changes in behavioral intentions translate into changes in on-the-road behavior.

\section{ACKNOWLEDGEMENTS}

Supported by a grant from General Motors.

\section{REFERENCES}

Goh, J., \& Wiegmann, D.A. (2001). Visual flight rules into instrument meteorological conditions: An empirical investigation of the possible causes. International Journal of Aviation Psychology, 11, 359-379.

Matsuura, T., Ishsida, T., Ishimatsu, K. (2002). Changes in seatbelt use after licensing: A developmental hypothesis for novice drivers. Transportation Research Part F: Traffic Psychology \& Behaviour, 5, 1-13.

Redelmeier, D. A., \& Tibshirani, R. J. (1997). Association between cellular-telephone calls and motor vehicle collisions. New England Journal of Medicine, 336, 453-458. 
Strayer, D. L., \& Johnston, W. A. (2001). Driven to distraction: Dual-task studies of simulated driving and conversing on a cellular phone. Psychological Science, 12, 462-466. 